\title{
ANIMAL HEALTH MONITORING WITH MISSING AND THEFT PREVENTION DEVICE USING WIRELESS SENSOR NETWORK AND INTERNET OF THINGS
}

\author{
Sundaramoorthi. P, \\ Department of Electrical and Electronics Engineering, \\ Nehru College of Engineering and Research Centre, \\ Pampady, Thrissur, Kerala, India \\ sundarped.sundar66@gmail.com \\ Rajeenamol. P.T, \\ Department of Electrical and Electronics Engineering, \\ Nehru College of Engineering and Research Centre, \\ Pampady, Thrissur, Kerala, India \\ rajeenamol2011@gmail.com \\ Anoopkumar. M.V, \\ Department of Electrical and Electronics Engineering, \\ Nehru College of Engineering and Research Centre, \\ Pampady, Thrissur, Kerala, India \\ anoopkumar.eee@ncerc.ac.in
}

Submitted: Mar, 04,2020 Revised: May, 14, 2020 Accepted: Jun, 04, 2020

\begin{abstract}
A device to know the health status of all the animals in a farm along with its missing and theft prevention is proposed in this article. The necessary sensors to sense the health information of animals and the short range wireless communication technology and internet to communicate with the owner of the farm, are planned to use in the devices. This system will be very helpful to diagnose the health condition of all the animals simultaneously and to improve it. The system can prevent the animals from missing or theft. A mobile application which acts as a user interface provides the owner with useful information such as animal's body temperature, heart rate, rumination and their presence inside the farm. The prototype of this idea is developed and is included for testing. Since the animal health status can be monitored from anywhere in world using a mobile application, the device will ease the work of farm owner and the laborers.
\end{abstract}

Keywords: animal health monitoring, internet of things, IoT, WSN, animal missing, theft prevention.

\section{INTRODUCTION}

Running animal farms and producing animal products is a big market all over the world. Continuous physical monitoring of the lots of animals which are available in farm is a tough job to the labors. Sometimes the diseases which are not notified may become life threatening. Kind of automated devices for monitoring the animals will ease the work of human labors. And also earlier prediction of diseases may save the animal's life. Various automated devices are introduced for the physiological monitoring of animals by different researchers in different timings. Various automated cattle health monitoring systems and its performances are studied in [1]. The author explains that, the data collecting from 
the automated devices helps the farm owners to make new strategies to improve the health status of animals and in turn increase the productivity.

The ZigBee wireless healthcare monitoring system is introduced in [2] for monitoring the heart rate, rumination and body temperature of the farm animal. The idea of wireless sensor network based monitoring of feeding and drinking behavior of cattle is discussed and simulated in [3]. Suggestions are described in the paper for better trade off between wireless devices to give better life time and less energy consumption.

Through automatically detecting the behavioral changes of pigs in piggeries, the pig's health status are monitored in [4]. It helps in early detection of health issues and reduces the impact on welfare.Continuous monitoring of electrocardiogram (ECG) and photoplethysmogram (PPG) of dogs is implemented in [5]. In this system, with the help of ECG and PPG sensor canine body area network is designed for behavioral study of dogs. By using a stainless steel roller ball nipple, the fluid intake behavior of animal is monitored in [6]. The system is actuated by animal's tongue and it is sensed with a 3 axis magnetometer. A special algorithm is developed for the respiratory analysis of dog in [7]. Sensor incorporated wearable device based network is created in [8], to locate and analyze the movement of wild animals. The difficulties in implementing this system and ideas for addressing the difficulties are explained.

Apart from sensors to measure physiological signals of animals, vision based animal behavior monitoring also plays major role in animal health monitoring. Individual pigs are localized, tracked and their behavioral metrics are studied in [9] with the help of an automated system which uses deep learning technique. The system is analyzing the images of pigs taken from camera. Vision based activity detection system is designed for mouse in [10]. Monocular images are used in this analysis. It used to detect the abnormalities in mouse behavior. Machine learning algorithm based study of animal behavior from video is discussed and evaluated in [11].

Nowadays, the Internet of Things (IoT) is available everywhere. In IoT any things can be connected to the internet cloud and can be accessed, monitored and controlled from anywhere in the world using internet enabled mobile phone or computer. Cattle health monitoring also started using IoT. Wireless body area network and IoT based dairy cow monitoring system is simulated in [12]. An IoT based prototype is developed in [13] using various sensors and Raspberry-Pi for monitoring the health of farm animals.

\section{PROPOSED SYSTEM}

The system proposed in this paper, is designed to monitor the physiological information of dairy cows and locate them using various sensors and IoT. The animal farm will consists of lots of animals. But the prototype is built and tested for minimum quantity. The heart rate, body temperature and rumination of each animal are monitored. Along with the body temperature sensor, the environmental temperature and humidity are measured to analyze the stress level of the animal. The Global Positioning System (GPS) transceiver fitted with each animal is used to find the position of the animal. This information will be useful to find the missing or theft animals. All the information measured using sensors are communicated to a central device which uploads the information in cloud storage. The mobile application software carried by farm supervisor or owner is helpful to collect and view the information from cloud. The view of system design is shown in Figure 1. 


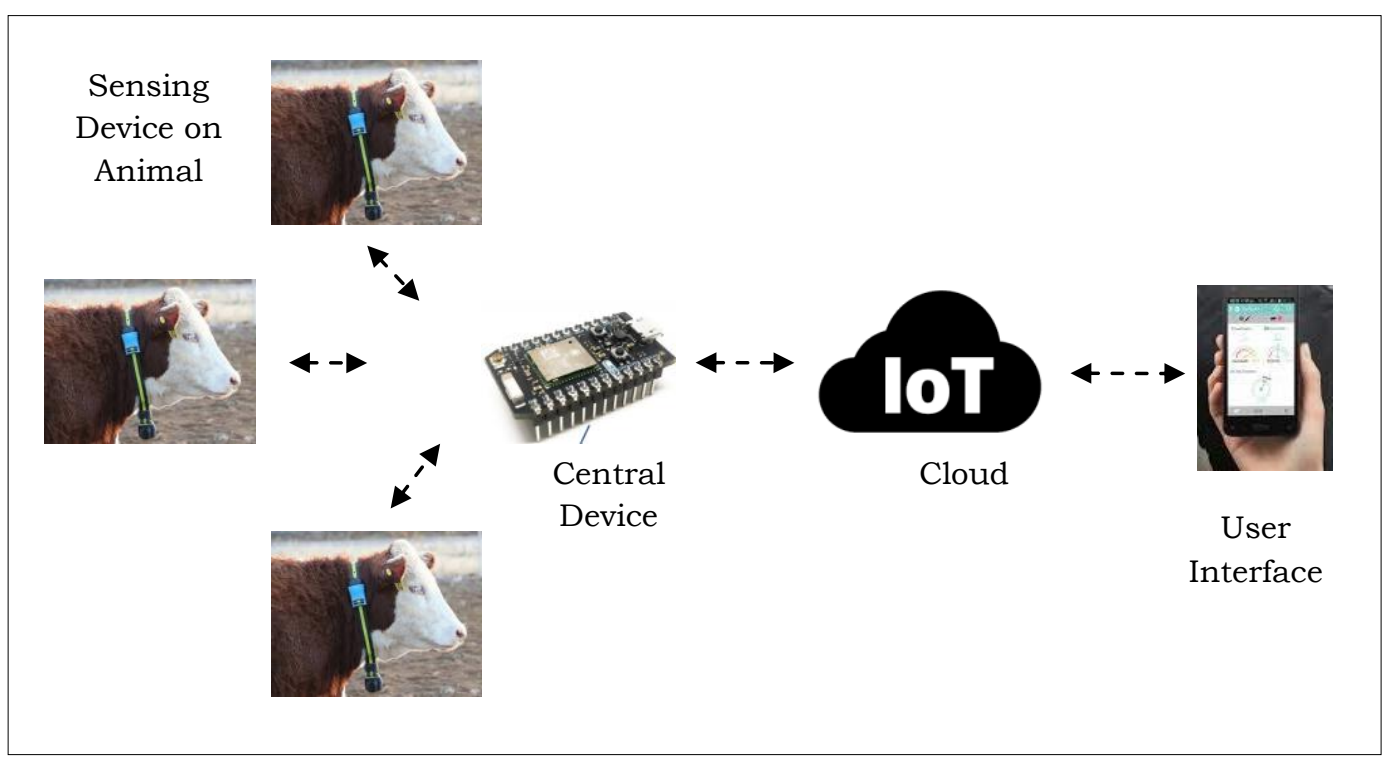

Fig. 1 View of system design

As shown in Figure 1, the overall system consists of multiples of sensing device which are fitted in animals, a central unit to collect the animal health information from various sensing devices, cloud storage and mobile application Wireless communication is established in between the sensing devices and central unit. Internet communication is used to upload the animal health information to cloud or receives from the cloud.

\section{Data Sensing Unit}

Data Sensing Unit (DSU) is the main part of animal health monitoring system. The block diagram of DSU is shown in Figure 2.

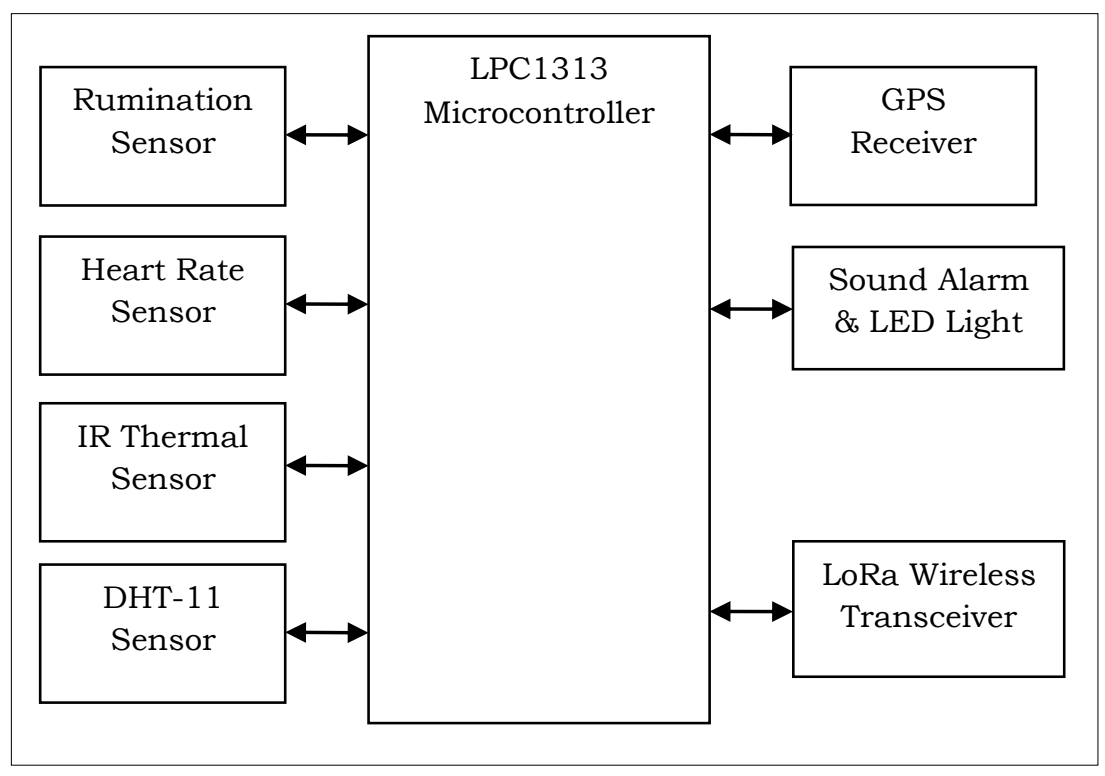

Fig. 2 Block diagram of DSU 
Each DSU consists of sensors to measure the health related information from the animal, where it is connected. The various sensors are rumination sensor, heart rate sensor, infrared (IR) based body temperature sensor, DHT 11 sensor to measure ambient temperature and humidity and GPS receiver to get location information of the animal.

The physical health of cow is directly related to its rumination or chewing [14][15]. In a day, a cow ruminate $500 \pm 100$ minutes on average. If it reduces, it indicates the cow is having health issue or it intake may be low. Finally it may affect the lactation. The 3 axis MEMS accelerometer is used to analyze the rumination pattern.

GPS transceiver receives the geographical position information such as latitude, longitude, altitude and time information from GPS satellites constellation. This information is used to track position of each animal. The device can be configured to auto mode using virtual fencing concept to alert in case of the animal is out of the boundary. Also when there is loss in wireless communication with particular sensing device, the central unit will send alert to the mobile application software. The geographical information of the farm's boundary is loaded in software to create the virtual fencing.

LoRa is an acronym for Long Range, is a spread spectrum modulation technique. LoRa wireless devices offers low power, secure and long range wireless communication. It is well suited for low cost and low power battery applications. Microchip LoRa device is used for this application. The Microcontroller processes the sensor information and communicates to the central unit through wireless medium.

\section{Central Unit}

The central unit consists of a Microcontroller along with LoRa device and WiFi communication modem. It relays the information to the cloud server using the internet connection available near to it. Figure 3 shows the block diagram of central unit.

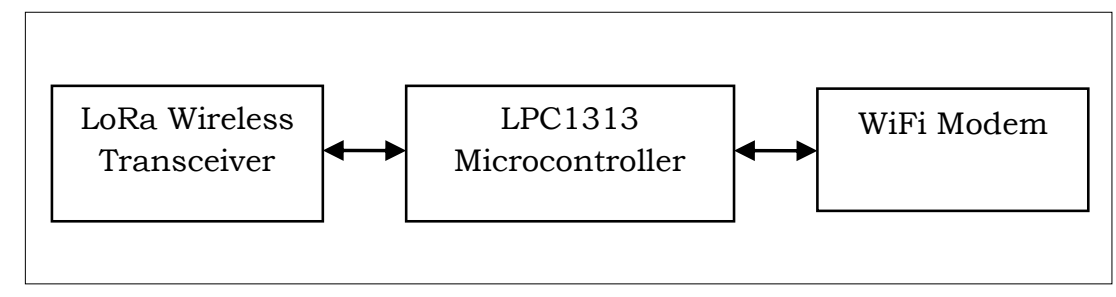

Fig. 3 Block diagram of Central Unit

\section{RESULTS AND DISCUSSION}

The objective of this work is to design an automated and continuous monitoring system, for the farm animals to detect its physiological health. By using the sensors, Microcontroller, LoRa wireless transceiver and internet communication a working model of the objective is implemented. Blynk IoT platform is used to connect the devices to cloud and monitor the data using mobile application. Blynk supports by providing secure cloud and freedom to design apps as per the monitoring and controlling requirements. It also helps to analyze the data using machine learning technique and to send email alert. The app interface is shown in Figure 4. 


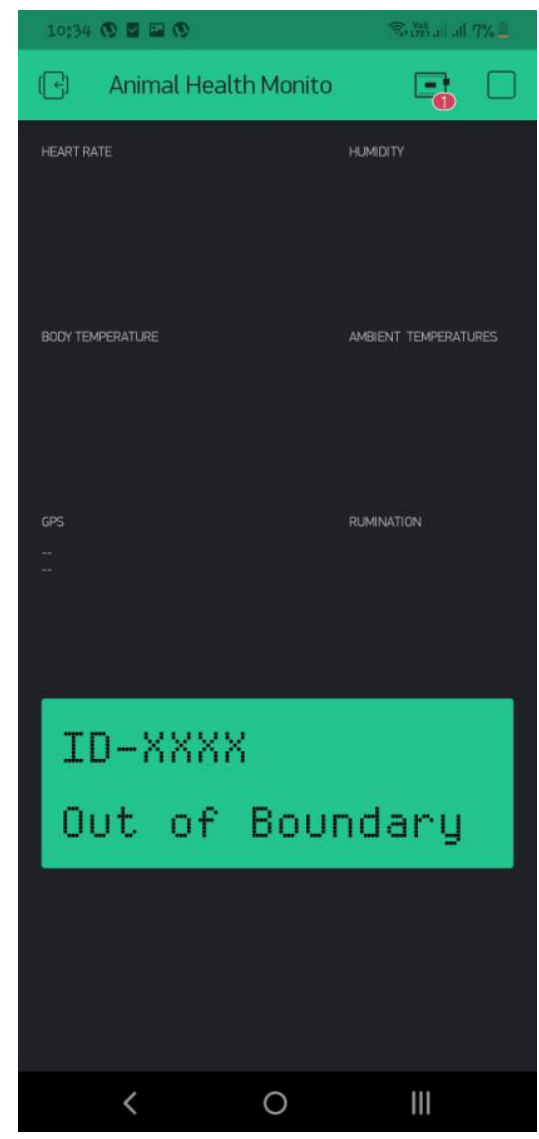

Fig. 4 Blynk's mobile user interface

The user interface shows the health parameters of animals to the farmers in their corresponding columns. It shows rumination, heart rate, GPS positions, body temperature, ambient temperature and humidity. Also it shows alerts in case of the abnormalities in any of these health parameters or when the animal is out of the boundary of farm.

\section{CONCLUSION}

The prototype of the animal health monitoring system is designed and implemented. The working model is tested and its performances are analyzed under various conditions. The model is working well and good. Monitoring of animals is very simple with the use of mobile application software. Since the animal's health information is available in online, the system gives the feature of anywhere anytime monitoring. It helps the farm owner for continuous monitoring of their farm animals. Earlier detection of diseases helps to treat the animal fast and it avoids loss of animal's health and unnecessary death. Theft or missing of animals can be avoided with the help of geographical positions of the animals. The LoRa wireless transceiver used for wireless communication between the sensing device and central unit gives long range wireless communication distance. In future, the wireless body area network can be implemented with more sensors, which will give detailed health information and will increase the productivity. 
Int. J.Adv.Sig.Img.Sci, Vol. 6, No. 1, 2020

\section{REFERENCES}

[1]. B. Sharma \& D. Koundal, "Cattle Health Monitoring System using Wireless Sensor Network: A Survey from Innovation Perspective", IET Wireless Sensor Systems, Vol. 8, No. 4, 2018, pp. 143-151.

[2]. A. Kumar \& G.P. Hancke, "A Zigbee-Based Animal Health Monitoring System”, IEEE Sensors Journal, Vol. 15, No. 1, 2014, pp. 610-617.

[3]. H. Wang, A.O. Fapojuwo \& R.J. Davies, "A Wireless Sensor Network for Feedlot Animal Health Monitoring", IEEE Sensors Journal, Vol. 16, No. 16, 2016, pp. 6433-6446.

[4]. S.G. Matthews, A.L. Miller, J. Clapp, T. Plötz \& I. Kyriazakis, "Early Detection of Health and Welfare Compromises Through Automated Detection of Behavioural Changes in Pigs", The Veterinary Journal, Vol. 217, 2016, pp. 43-51.

[5]. R. Brugarolas, T. Latif, J. Dieffenderfer, K. Walker, S. Yuschak, B.L. Sherman, ... \& A. Bozkurt, "Wearable Heart Rate Sensor Systems for Wireless Canine Health Monitoring", IEEE Sensors Journal, Vol. 16, No. 10, 2015, pp. 3454-3464.

[6]. S. Sargolzaei, H. Elahi, A. Sokoloff \& M. Ghovanloo, "A Dual-Mode Magnetic-Acoustic System for Monitoring Fluid Intake Behavior in Animals", IEEE Transactions on Biomedical Engineering, Vol. 64, No. 9, 2016, pp. 2090-2097.

[7]. C.H. Antink, M. Pirhonen, H. Väätäjä, S. Somppi, H. Törnqvist, A.V. Cardó, ... \& A. Vehkaoja "Sensor Fusion for Unobtrusive Respiratory Rate Estimation in Dogs", IEEE Sensors Journal, Vol. 19, No. 16, 2019, pp. 7072-7081.

[8]. L. Camal, A. Kirtane, T. Blanco, R. Casas, F. Rossano \& B. Aksanli, "A Wearable Device Network to Track Animal Behavior and Relationships in the Wild", In IEEE 10th Annual Ubiquitous Computing, Electronics \& Mobile Communication Conference (UEMCON), 2019, pp. 0198-0202.

[9]. J. Cowton, I. Kyriazakis \& J. Bacardit, "Automated Individual Pig Localisation, Tracking and Behaviour Metric Extraction Using Deep Learning", IEEE Access, Vol. 7, 2019, pp. 108049-108060.

[10]. G. Salem, J. Krynitsky, M. Hayes, T. Pohida \& X. Burgos-Artizzu, "ThreeDimensional Pose Estimation for Laboratory Mouse from Monocular Images”, IEEE Transactions on Image Processing, Vol. 28, No. 9, 2019, pp. 4273-4287.

[11]. T. Manning, M. Somarriba, R. Roehe, S. Turner, H. Wang, H. Zheng,... \& P. Walsh, "Automated Object Tracking for Animal Behaviour Studies", In IEEE International Conference on Bioinformatics and Biomedicine (BIBM), 2019, pp. 1876-1883.

[12]. S. Benaissa, D. Plets, E. Tanghe, J. Trogh, L. Martens, L. Vandaele, ... \& W. Joseph, "Internet of Animals: Characterisation of LoRa sub-GHz off-body Wireless Channel in Dairy Barns", Electronics Letters, Vol. 53, No. 18, 2017, pp. 1281-1283.

[13]. S. Kumari \& S.K. Yadav, "Development of IoT Based Smart Animal Health Monitoring System Using Raspberry Pi", International Journal of Advanced Studies of Scientific Research, Vol. 3, No. 8, 2018, pp. 24-31. 
[14]. H. Wu, F. Bai, Z. Zhong, X. Cheng \& D. Fan, "Research on Cow Rumination Monitoring Based on New Activity Sensor". In IEEE 9th International Conference on Information Science and Technology (ICIST), 2019, pp. 199-204.

[15]. https://www.allflex.global/rumination-monitoring-helps-livestock-healthmanagement/ 